\title{
Synthesis and Detailed Spectroscopic Characterization of Two Novel $N$-(3-Acetyl- 2-thienyl)acetamides
}

\section{Gernot A. Eller* and Wolfgang Holzer}

Department of Drug Synthesis, Faculty of Life Sciences, University of Vienna, Althanstrasse 14, A-1090 Vienna, Austria

Phone: +43-1-4277-55634,

Email: gernot.eller@univie.ac.at

* Author to whom correspondence should be addressed

Received: 1 September 2006 / Accepted: 20 October 2006 / Published: 1 December 2006

Keywords: $N$-acylations, 3 -acetylthiophen-2-amine, NMR spectroscopy

\section{Abstract:}

The title compounds - N-(3-acetyl-2-thienyl)-2-bromoacetamide and $\mathrm{N}$-(3-acetyl-2-thienyl)-2phthalimidoacetamide - were synthesized in one step from 3-acetylthiophen-2-amine and the corresponding acetyl halogenides. Detailed spectroscopic data $\left({ }^{1} \mathrm{H} N \mathrm{NR},{ }^{13} \mathrm{C} \mathrm{NMR},{ }^{15} \mathrm{~N} \mathrm{NMR}, \mathrm{MS}, \mathrm{IR}\right)$ for these compounds are presented.

Recently, we have investigated a modified Gewald reaction [1] for the preparation of 3-acetyl2-aminothiophenes [2]. We here report the synthesis of two acetamides derived from 3-acetylthiophen2-amine (1) (Scheme 1). These molecules are expected to be versatile intermediates for advanced investigations regarding the chemistry of 3-acetylthiophenes of type $\mathbf{1}$.

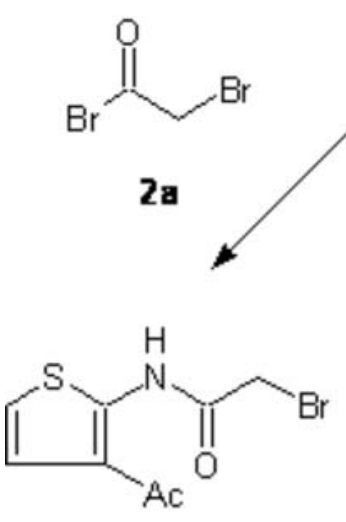

3a<smiles>Nc1sccc1Cl</smiles>

1<smiles>O=C(Cl)CN1C(=O)c2ccccc2C1=O</smiles><smiles>N#Cc1ccsc1NC(=O)CN1C(=O)c2ccccc2C1=O</smiles>

$3 \mathbf{b}$

Scheme 1. Preparation of the title compounds $3 a$ and $3 b$

\section{$N$-(3-Acetyl-2-thienyl)-2-bromoacetamide (3a):}

Under stirring at room temperature, to $4.23 \mathrm{~g}(30 \mathrm{mmol})$ thiophenamine 1 [2] in $70 \mathrm{~mL}$ of dry 1,4-dioxane

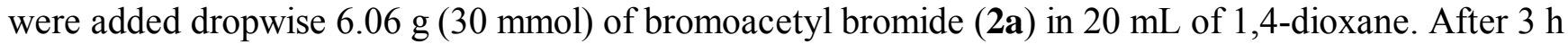
the reaction mixture was poured into ice-cold $\mathrm{H}_{2} \mathrm{O}(\mathrm{ca} .300 \mathrm{~mL})$, the resulting precipitate was filtered off, washed with $\mathrm{H}_{2} \mathrm{O}$, and dried under reduced pressure to afford pure $3 \mathbf{a}(4.72 \mathrm{~g}, 60 \%)$ as a beige powder. The compound slowly decomposes in DMSO- or MeOH-solution.

Melting point: $96-97^{\circ} \mathrm{C}$. 
IR (KBr) [3]: 1660, $1640 \mathrm{~cm}^{-1}$.

${ }^{1} \mathrm{H}$ NMR (300 MHz, DMSO-d6) [4]: $\delta$ (ppm) 12.18 (s, 1H, NH), $7.43\left(\mathrm{~d},{ }^{3} J(\mathrm{H} 4, \mathrm{H} 5)=5.8 \mathrm{~Hz}, 1 \mathrm{H}, \mathrm{H} 4\right)$, $7.06\left(\mathrm{~d},{ }^{3} \mathrm{~J}(\mathrm{H} 4, \mathrm{H} 5)=5.8 \mathrm{~Hz}, 1 \mathrm{H}, \mathrm{H} 5\right), 4.43\left(\mathrm{~s}, 2 \mathrm{H}, \mathrm{CH}_{2}\right), 2.52\left(\mathrm{~s}, 3 \mathrm{H}, \mathrm{CH}_{3}\right)$.

${ }^{1} \mathrm{H}$ NMR (500 MHz, CDCl 3 ) [5]: $\delta(\mathrm{ppm}) 12.66$ (s, 1H, NH), $7.23\left(\mathrm{~d},{ }^{3} J(\mathrm{H} 4, \mathrm{H} 5)=5.8 \mathrm{~Hz}, 1 \mathrm{H}, \mathrm{H} 4\right), 6.81$ $\left(\mathrm{d},{ }^{3} J(\mathrm{H} 4, \mathrm{H} 5)=5.8 \mathrm{~Hz}, 1 \mathrm{H}, \mathrm{H} 5\right), 4.09\left(\mathrm{~s}, 2 \mathrm{H}, \mathrm{CH}_{2}\right), 2.55\left(\mathrm{~s}, 3 \mathrm{H}, \mathrm{CH}_{3}\right)$.

${ }^{13} \mathrm{C}$ NMR (75 MHz, DMSO-d $)$ [4]: $\delta(\mathrm{ppm}) 195.8\left(\mathrm{COCH}_{3}\right), 164.6\left(\mathrm{NCO},{ }^{2} J\left(\mathrm{NCO}, \mathrm{CH}_{2}\right)=4.3 \mathrm{~Hz}\right.$, $\left.{ }^{2} J(\mathrm{NCO}, \mathrm{NH})=4.3 \mathrm{~Hz}\right), 147.0(\mathrm{C} 2), 125.2\left(\mathrm{C} 4,{ }^{1} \mathrm{~J}=170.2 \mathrm{~Hz},{ }^{2} \mathrm{~J}(\mathrm{C} 4, \mathrm{H} 5)=4.2 \mathrm{~Hz}\right), 121.8(\mathrm{C} 3), 117.1$ $\left(\mathrm{C} 5,{ }^{1} J=189.3 \mathrm{~Hz},{ }^{2} J(\mathrm{C} 5, \mathrm{H} 4)=6.0 \mathrm{~Hz}\right), 29.0\left(\mathrm{CH}_{2},{ }^{1} J=155.6 \mathrm{~Hz}\right), 28.8\left(\mathrm{CH}_{3},{ }^{1} J=127.7 \mathrm{~Hz}\right)$.

${ }^{13} \mathrm{C} \mathrm{NMR}\left(125 \mathrm{MHz}, \mathrm{CDCl}_{3}\right)[5]: \delta(\mathrm{ppm}) 196.0\left(\mathrm{COCH}_{3},{ }^{2} J\left(\mathrm{COCH}_{3}, \mathrm{CH}_{3}\right)=5.9 \mathrm{~Hz},{ }^{3} J(\mathrm{CO}, \mathrm{H} 4)=0.9\right.$ $\mathrm{Hz}), 164.1\left(\mathrm{NCO},{ }^{2} J\left(\mathrm{NCO}, \mathrm{CH}_{2}\right)=4.5 \mathrm{~Hz}\right), 148.3\left(\mathrm{C} 2,{ }^{2} J(\mathrm{C} 2, \mathrm{NH})=2.1 \mathrm{~Hz},{ }^{3} J(\mathrm{C} 2, \mathrm{H} 4)=10.0 \mathrm{~Hz}\right.$, $\left.{ }^{3} J(\mathrm{C} 2, \mathrm{H} 5)=7.6 \mathrm{~Hz}\right), 124.4\left(\mathrm{C} 4,{ }^{1} J=168.8 \mathrm{~Hz},{ }^{2} \mathrm{~J}(\mathrm{C} 4, \mathrm{H} 5)=3.6 \mathrm{~Hz}\right), 122.0\left(\mathrm{C} 3,{ }^{2} \mathrm{~J}(\mathrm{C} 3, \mathrm{H} 4)=5.8 \mathrm{~Hz}\right.$, $\left.\left.{ }^{3} \mathrm{~J}(\mathrm{C} 3, \mathrm{H} 5)=9.1 \mathrm{~Hz}\right),{ }^{3} \mathrm{~J}\left(\mathrm{C} 3, \mathrm{CH}_{3}\right)=1.3 \mathrm{~Hz}\right), 116.9\left(\mathrm{C} 5,{ }^{1} \mathrm{~J}=187.7 \mathrm{~Hz},{ }^{2} \mathrm{~J}(\mathrm{C} 5, \mathrm{H} 4)=5.0 \mathrm{~Hz}\right), 28.7\left(\mathrm{CH}_{3}\right.$, $\left.{ }^{1} J=127.9 \mathrm{~Hz}\right), 27.9\left(\mathrm{CH}_{2},{ }^{1} J=153.9 \mathrm{~Hz}\right)$.

${ }^{15} \mathrm{~N}$ NMR $\left(50 \mathrm{MHz}, \mathrm{CDCl}_{3}\right)[6]: \delta(\mathrm{ppm})-248.9(\mathrm{NH})$.

$\operatorname{MS}(\mathrm{m} / \mathrm{z}, \%)$ [7]: $263\left(\mathrm{M}^{+}, 27\right), 261\left(\mathrm{M}^{+}, 25\right), 141(100), 126(75), 43(59)$.

Elemental Analysis: Calculated for $\mathrm{C}_{8} \mathrm{H}_{8} \mathrm{BrNO}_{2} \mathrm{~S}(262.12) \cdot 0.1 \mathrm{H}_{2} \mathrm{O}: \mathrm{C}, 36.41 \%$; H, 3.13\%; N, 5.31\%. Found: C, 36.15\%; H, 2.92\%; N, 5.03\%.

\section{$\mathrm{N}$-(3-Acetyl-2-thienyl)-2-(1,3-dioxo-1,3-dihydro-2H-isoindol-2-yl)acetamide (3b):}

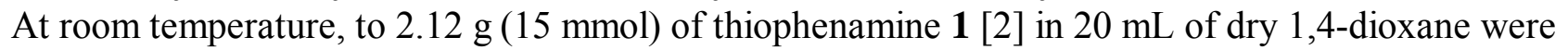
added dropwise $3.35 \mathrm{~g}$ (15 mmol) of phthalimidoacetyl chloride (2b) [8] in $20 \mathrm{~mL}$ of 1,4-dioxane. The reaction mixture was stirred overnight and then poured into $\mathrm{H}_{2} \mathrm{O}$ (ca. $100 \mathrm{~mL}$ ). Upon neutralization with solid $\mathrm{NaHCO}_{3}$ a yellowish precipitate was formed which was filtered off, washed with $\mathrm{H}_{2} \mathrm{O}$, and dried under reduced pressure to afford pure $3 \mathbf{b}(4.33 \mathrm{~g}, 88 \%)$ as a yellowish powder.

Melting point: $208-212^{\circ} \mathrm{C}$.

IR (KBr) [3]: 1773, 1719, 1693, $1635 \mathrm{~cm}^{-1}$.

${ }^{1} \mathrm{H}$ NMR (500 MHz, $\mathrm{CDCl}_{3}$ ) [5]: $\delta(\mathrm{ppm}) 12.25$ (s, 1H, NH), 7.91 (m, 2H, Phth-H3,6), 7.76 (m, 2H, Phth-H4,5), 7.17 (d, ${ }^{3} J\left(\right.$ Th-H4,Th-H5) $=5.8 \mathrm{~Hz}, 1 \mathrm{H}$, Th-H4), $6.75\left(\mathrm{~d},{ }^{3} J(\mathrm{Th}-\mathrm{H} 5, \mathrm{Th}-\mathrm{H} 4)=5.8 \mathrm{~Hz}, 1 \mathrm{H}\right.$, Th-H5), 4.65 (s, 2H, $\left.\mathrm{CH}_{2}\right), 2.49\left(\mathrm{~s}, 3 \mathrm{H}, \mathrm{CH}_{3}\right)$.

${ }^{13} \mathrm{C}$ NMR (125 MHz, $\mathrm{CDCl}_{3}$ ) [5]: $\delta$ (ppm) $196.1\left(\mathrm{COCH}_{3}\right), 167.4$ (Phth-CO), 164.1 (HNCO), 148.5 (Th-C2), 134.4 (Phth-C4,5), 131.9 (Phth-C1,2), 124.2 (Th-C4), 123.8 (Phth-C3,6), 121.5 (Th-C3), 116.6 (Th-C5), $40.7\left(\mathrm{CH}_{2}\right), 28.6\left(\mathrm{CH}_{3}\right)$.

${ }^{15} \mathrm{~N}$ NMR $\left(50 \mathrm{MHz}, \mathrm{CDCl}_{3}\right)[6]: \delta(\mathrm{ppm})-228.8\left(\mathrm{NCH}_{2}\right),-252.7(\mathrm{NH})$.

$\operatorname{MS}(\mathrm{m} / \mathrm{z}, \%)[7]: 328\left(\mathrm{M}^{+}, 17\right), 168(17), 160$ (100), $141(27)$. 
Elemental Analysis: Calculated for $\mathrm{C}_{16} \mathrm{H}_{12} \mathrm{~N}_{2} \mathrm{O}_{4} \mathrm{~S}(328.34) \cdot 0.1 \mathrm{H}_{2} \mathrm{O}$ : C, 58.21\%; H, 3.72\%; N, 8.49\%. Found: C, $57.86 \%$; H, 3.87\%; N, 8.40\%.

\section{References and Notes}

1. Gewald, K. Chem. Ber. 1965, 98, 3571-3577.

2. Eller, G. A.; Holzer, W. Molecules 2006, 11, 371-376.

3. The spectrum was obtained on a Perkin-Elmer FTIR 1605 spectrophotometer.

4. The spectrum was obtained on a Varian UnityPlus 300 spectrometer $\left(299.95 \mathrm{MHz}\right.$ for ${ }^{1} \mathrm{H}, 75.43 \mathrm{MHz}$ for ${ }^{13} \mathrm{C}$ ) at $28^{\circ} \mathrm{C}$. The center of the solvent signal was used as an internal standard which was related to TMS with $\delta 2.49 \mathrm{ppm}\left({ }^{1} \mathrm{H}\right.$ NMR $)$ and $\delta 39.5 \mathrm{ppm}\left({ }^{13} \mathrm{C} \mathrm{NMR}\right)$.

5. The spectrum was obtained on a Bruker Avance 500 spectrometer $\left(500.13 \mathrm{MHz}\right.$ for ${ }^{1} \mathrm{H}, 125.77 \mathrm{MHz}$ for ${ }^{13} \mathrm{C}$ ) at $294 \mathrm{~K}$. The center of the solvent signal was used as an internal standard which was related to TMS with $\delta 7.26 \mathrm{ppm}\left({ }^{1} \mathrm{H}\right.$ NMR $)$ and $\delta 77.0 \mathrm{ppm}\left({ }^{13} \mathrm{C} \mathrm{NMR}\right)$.

6. The spectrum was obtained on a Bruker Avance 500 spectrometer $\left(50.68 \mathrm{MHz}\right.$ for $\left.{ }^{15} \mathrm{~N}\right)$ and was referenced against neat, external nitromethane (coaxial capillary).

7. The spectrum was obtained on a Shimadzu QP 1000 instrument (EI, 70eV).

8. Usifoh, C. O.; Lambert, D. M.; Wouters, J.; Scriba, G. K. E. Arch. Pharm. (Weinheim, Ger.) 2001, 334, 323-331.

(C) 2006 MDPI. All rights reserved. 